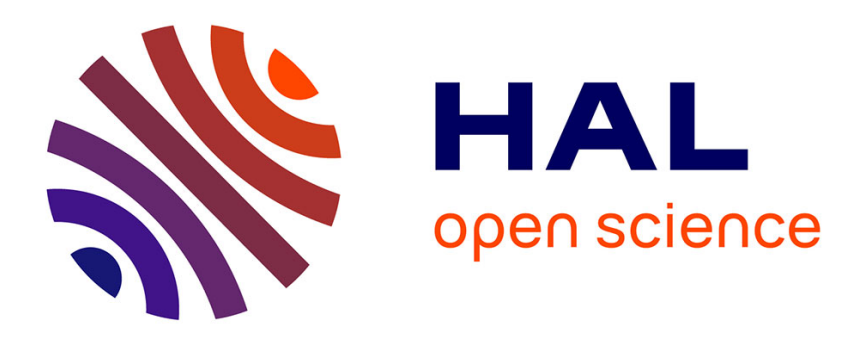

\title{
Architecture ordinaire et hommes pluriels
}

\author{
Jean-Baptiste Minnaert
}

\section{To cite this version:}

Jean-Baptiste Minnaert. Architecture ordinaire et hommes pluriels. Ligeia, dossiers sur l'art, 2009, L'autre Europe: art d'avant-garde et identité culturelle, 93-96, pp.38-44. halshs-01176314

\section{HAL Id: halshs-01176314 https://shs.hal.science/halshs-01176314}

Submitted on 15 Jul 2015

HAL is a multi-disciplinary open access archive for the deposit and dissemination of scientific research documents, whether they are published or not. The documents may come from teaching and research institutions in France or abroad, or from public or private research centers.
L'archive ouverte pluridisciplinaire HAL, est destinée au dépôt et à la diffusion de documents scientifiques de niveau recherche, publiés ou non, émanant des établissements d'enseignement et de recherche français ou étrangers, des laboratoires publics ou privés.

\section{()ㅜ) $\Theta$}

Distributed under a Creative Commons Attribution - NoDerivatives| 4.0 International 


\title{
ARCHITECTURE ORDINAIRE ET HOMMES PLURIELS
}

\author{
Jean-Baptiste Minnaert
}

"Plus les acteurs sont le produit de formes de vie sociales hétérogènes, voire contradictoires, plus la logique de la situation présente joue un rôle central dans la réactivation d'une partie des expériences passées incorporées. Le passé est donc 'ouvert' différemment selon la nature et la configuration de la situation présente."1

L'histoire de l'architecture contemporaine s'est construite sur des centrages occidentaux et sur des formes de récit qui font la part belle au créateur et à l’innovation, parfois au détriment du collectif et de l'ordinaire, le plus souvent aux dépens de l'appréhension de différentes temporalités, en particulier la longue durée. Cela génère bien des impensés qui subsistent dans les lignes de force actuelles du champ disciplinaire, que ce soit de manière explicite par le choix des objets et des méthodes, ou de manière implicite par la rémanence d'habitudes de pensée anciennes, et en raison de la forte - et souvent salutaire - inertie des corpus constitués et des historiographies.

\section{Cadres institutionnels et théoriques}

En France, l'étude des productions théoriques et bâties de la période contemporaine en matière d'architecture et de formes urbaines est menée à l'université, dans les départements d'histoire de l'art, ainsi que dans les départements d'histoire, de philosophie, d'arts plastiques, de littérature, de sociologie, d'anthropologie, d'ethnologie, de géographie, d'urbanisme. Elle est pratiquée aussi par quelques musées et par quelques équipes du CNRS, ainsi que dans les écoles d'architecture et, dans une moindre mesure, dans les écoles des Beaux-Arts. Cette dispersion est la condition d'une indispensable et féconde pluralité d'approches, mais les questions touchant à la théorie et à l'histoire de l'histoire de l'architecture contemporaine méritent aussi d'être débattues au sein de structures de recherche spécifiques².

À cette dispersion se superpose la disparité des objectifs intellectuels et socioprofessionnels que s'assignent les historiens d'architecture, dans leurs activités souvent

\footnotetext{
1. Bernard Lahire, L'homme pluriel. Les ressorts de l'action, Amrand Colin / Nathan, Paris, 2001 (édition consultée : Hachette Littérature), p. 87.

2. Ces questions sont formulées ainsi dans le programme de recherche de l'InTRu (Interactions, Transferts, Ruptures artistiques et culturels), Jeune équipe créée en 2008 à l'université François Rabelais de Tours. Les lignes qui suivent se réfèrent au programme scientifique de l'Intru, rédigé par Éric de Chassey, par France Nerlich et par nous-même, et en développent quelques thèmes, plus directement liés à l'histoire de l'architecture contemporaine.
} 
parallèles de chercheur, d'expert et de critique. Rarement s'interroge-t-on sur les porosités, les sauts d'échelle, les porte-à-faux éthiques, les hiatus méthodologiques, notamment les conflits de tempos opérationnels, qu'entraîne le passage de l'exercice de la recherche fondamentale à ses champs d'application que sont l'expertise et la critique. On aurait tort de voir ces deux dernières activités uniquement comme des sous-produits intellectuels. Nombre d'objets scientifiques aujourd'hui reconnus se sont agrégés sur les chemins de traverse de l'expertise et de la critique, dans l'empirisme et l'urgence de réponses scientifiquement légitimées à apporter à des questionnements de la société, du politique ou du marché. Les jeux croisés de l'expertise et de la critique avec la recherche fondamentale constituent un axe important de l'historiographie de l'histoire de l'architecture, et aussi l'un de ses principaux angles morts ${ }^{3}$.

Sur la matière même de l'histoire et sur ses méthodes, il serait tout aussi utile de mener quelques réflexions. À l'instar de l'histoire de l'art en général, l'histoire de l'architecture contemporaine se contente souvent de tracer la circulation des hommes, des idéologies, des doctrines, des modèles, des types et des savoir-faire, d'en construire la généalogie et d'en préciser les déclinaisons et les réceptions, ainsi que les transpositions et trahisons, souvent à travers le cas particulier d'un concepteur, d'un espace urbanisé ou d'un type construit. Ce genre d'approche peut être cependant enrichi par des outils et des méthodes qui permettent de penser sur d'autres échelles de temps et d'espace, sur d'autres lignes intellectuelles. Ainsi pourrait-on s'interroger sur la façon dont ces objets peuvent être situés sur la trame d'interactions mises ou non en système, étant à la fois supports et substrats de processus de transferts, d'interactions et de ruptures. Il serait utile à cet égard de réinvestir les théories de la réception (Hans Robert Jauss ${ }^{4}$ et Wolfgang Iser), de la rupture (Michel Foucault), des transferts culturels (Michael Werner et Michel Espagne ${ }^{5}$ ), de l'influence (Harold Bloomº), voire de la transtextualité (Gérard Genette ${ }^{7}$ ). La théorie de l'archéologie générale (Philippe Bruneau et Pierre-Yves Balut ${ }^{8}$ ) est une grille de lecture particulièrement féconde, autant que certaines théories de l'action (Michel Crozier et Erhard Friedberg9, Bernard Lahire) et, last but not least, les travaux fondateurs de Maurice Halbwachs ${ }^{10}$, que les historiens d'architecture utilisent sans doute avec trop de parcimonie.

\footnotetext{
${ }^{3}$. Nous avons abordé ces questions dans Jean-Baptiste Minnaert (dir.), Histoires d'architectures en Méditerranée XIXe-XXe siècles. Écrire l'histoire d'un héritage bâti, Éditions de la Villette, Paris, 2005, ainsi que dans un numéro thématique, sous notre direction, de la revue Histoire de l'art, "Nouvelles approches en architecture", n 59, octobre 2006.

${ }^{4}$. Hans Robert Jauss, Pour une esthétique de la réception, Gallimard, Paris, 1978.

5 . Michel Espagne et Michael Werner (dir.), Transferts. Les relations interculturelles dans l'espace franco-allemand XVIIIeXIXe siècle), Éditions Recherches sur les civilisations, Paris, 1988.

6. Harold Bloom, The Anxiety of Influence, a Theory of Poetry, Oxford University Press, Londres, 1975.

7. Gérard Genette, Figures III, Paris, Éditions du Seuil, 1972; Gérard Genette, Palimpsestes, La littérature au second degré, Éditions du Seuil, Paris, 1982.

${ }^{8}$ Philippe Bruneau, Pierre-Yves Balut, Artistique et archéologie, Presses de l'université Paris-Sorbonne, Paris, 1997.

9. Michel Crozier, Erhard Friedberg, L'acteur et le système. Les contraintes de l'action collective, Éditions du Seuil, Paris, 1977.
} 
S’il est entré dans la vulgate que l'historien "invente" ses objets, les historiens de l'art prennent-ils toute la mesure du statut culturellement construit de leurs sources et de leurs objets, repérés et validés, puis mis dans l'ordre de l'intelligibilité historiographique, connectés enfin avec les différents champs du savoir ${ }^{11}$ ? Ainsi la singularité des productions et des trajectoires créatives et intellectuelles pourrait-elle être appréhendée à travers les concepts d'échelle d'observation et de jeux d'échelles ${ }^{12}$. Les objets ainsi étudiés pourraient être traités non seulement dans leurs rapports à toutes sortes d'agrégats construits par l'histoire, mais aussi dans une posture de micro-histoire et suivant des temporalités multiples, non pas seulement superposées, mais plus généralement entrecroisées, dans tous les cas discontinues. Si le choix d'une échelle d'analyse est obligatoire dans toute opération historienne, encore faut-il que celle-ci soit définie et articulée avec d'autres échelles $^{13}$. À ce titre, on pourrait s'interroger sur le fait que l'appréhension du changement soit l'une des principales modalités de validation des méthodes et des théories de l'histoire ${ }^{14}$. Sans doute, l'appréhension parallèle des permanences situerait mieux les objets étudiés. Enfin confond-on trop souvent les échelles de temps et d'espace auxquelles est situé l'objet, et les échelles auxquelles se situent les analyses que l'historien lui consacre à un moment donné.

Affirmons d'entrée de jeu qu'il n'est ici nulle tentation d'esquisser une grille d'interprétation euclidienne et unificatrice, ce qui serait aussi naïf que vain. Les savoirs et leurs théorisations constituent d'irréductibles hétérotopies ${ }^{15}$. Les discontinuités ne se limitent nullement aux données initiales, que l'opération historiographique devrait réduire à une forme plus ou moins stable d'unité. Les discontinuités sont présentes aussi dans le rendu final des résultats de l'analyse, à tout le moins dans leur mise en rapport avec les savoirs déjà constitués. Ces discontinuités ne sont pas pour autant des symptômes d'impuissance théorique ou opérationnelle. Elles sont bien au contraire une forme de potentiel interrogeable, voire exploitable à tous stades de l'opération historiographique.

Il est par ailleurs utile de s'interroger sur les contingences rédactionnelles de l'écriture de l'histoire. La forme du texte narratif, utilisée par les écrivains comme par les historiens, impose sa propre diachronie, qui n'est jamais celle des faits que l'historien a assemblés en démonstration. À l'évidence, ces faits peuvent être synchroniques, ou relevant de temporalités très complexes que,

\footnotetext{
${ }^{10}$. En particulier Maurice Halbwachs, Les cadres sociaux de la mémoire, 1925, Albin Michel, Paris, 1994.

11. Paul Ricoeur, La mémoire, l'bistoire, l'oubli, Éditions du Seuil, Paris, 2000, p. 169-174.

12. Jacques Revel (dir.), Jeux d'échelles. La micro-analyse à l'expérience, Gallimard / Seuil, Paris, 1996. Voir en particulier l'article de Bernard Lepetit, "De l'échelle en histoire", p. 71-93.

13. Bernard Lepetit, id., p. 86.

14. "L'adoption d'une échelle est d'abord le choix d'un point de vue de connaissance". Bernard Lepetit, in Jacques Revel (dir.), op. cit., p. 80.

15. Michel Foucault, Les mots et les choses. Une archéologie des sciences humaines, Gallimard, Paris, 1966. Michel Foucault, L'archéologie du savoir, Paris, Gallimard, 1969.
} 
cependant, lisse l'écriture. Comme le remarque Sabina Loriga ${ }^{16}$, Thomas Carlyle en son temps réfléchissait déjà pour l'histoire à une écriture tridimensionnelle, qu'André Gide au demeurant pratiqua plusieurs décennies plus tard avec Les faux-monnayeurs.

Quelle histoire de quel art souhaite-t-on écrire, à quel horizon culturel, à quelle amplitude géographique, dans quelles temporalités, dans quels rapports à l'historiographie de l'objet et à l'historiographie des méthodes qui l'appréhendent? Repenser les fondements de cette hiérarchie et des structures historiographiques permettrait de tenir compte des modifications de perception et de réception qui font de l'histoire de l'art, comme des autres sciences de l'homme et de la société, une discipline à la fois inerte et labile, en déplacement incessant. Nos interrogations gravitent autour de l'invention permanente de nouveaux objets et sources, de l'incessante réélaboration des outils et des catégories d'analyse, du questionnement permanent des idéologies qui travaillent, parfois sourdement, la réflexion historienne et le métier d'historien ${ }^{17}$.

\section{De l'ordinaire à l'exceptionnel : hiérarchiser les objets et les méthodes}

"En général l'histoire des idées traite le champ des discours comme un domaine à deux valeurs ; tout élément qu'on y repère peut être caractérisé comme ancien ou nouveau ; inédit ou répété, traditionnel ou original ; conforme à un type moyen ou déviant. On peut donc distinguer deux catégories de formulations; celles, valorisées et relativement peu nombreuses, qui apparaissent pour la première fois, qui n'ont pas d'antécédents semblables à elles, qui vont éventuellement servir de modèles aux autres, et qui dans cette mesure méritent de passer pour des créations ; et celles, banales, quotidiennes, massives, qui ne sont pas responsables d'ellesmêmes et qui dérivent, parfois pour le répéter textuellement, de ce qui a été déjà dit. [...] Dans le premier cas, l'histoire des idées décrit une succession d'événements de pensée; dans le second, on a des nappes ininterrompues d'effets. [...] Le problème est alors de retrouver le point de rupture, d'établir, avec le plus de précision possible, le partage entre l'épaisseur implicite du déjàlà, la fidélité peut-être involontaire à l'opinion acquise, la loi des fatalités discursives, et la vivacité de la création, le saut dans l'irréductible différence."18

Les nouveaux corpus et les nouvelles méthodes qui qualifient l'ordinaire et soulèvent la question du quantitatif ont fait voler en éclats le primat des généalogies fonctionnalistes et formalistes que l'histoire de l'art a construites au milieu du XXe siècle. Près de quatre-vingts ans après la fondation des Annales $^{19}$, on constate que l'histoire de l'art a fini par intérioriser peu ou

\footnotetext{
16. Sabina Loriga, "La biographie comme problème”, in Jacques Revel (dir.), op. cit., p. 209-231.

17. François Bédarida (dir.), L’histoire et le métier d'historien en France, 1945-1995, Éditions de la Maison des sciences de l'homme Paris, Paris, 1995. Voir aussi Antoine Prost, Douze leçons sur l'histoire, Éditions du Seuil, Paris, 1996.

18. Michel Foucault, L’archéologie du savoir, Gallimard, Paris, 1969 (édition consultée: Tel Gallimard), p. 191-193.

19. André Burguière, L'école des Annales. Une histoire intellectuelle, Odile Jacob, Paris, 2006.
} 
prou le concept “d'histoire-problème”. Elle ne s'est guère employée pour autant à penser systématiquement la tension entre l'ordinaire et l'exceptionnel, tant pour les objets que pour les méthodes, ni à en tirer des conséquences d'ordre épistémologique, ni a fortiori à construire explicitement de nouvelles représentations d'elle-même.

L'architecture en masse et les ensembles urbains que les processus de patrimonialisation signalent à l'attention des chercheurs depuis près d'un demi-siècle ne peuvent pas être rangés dans les catégories esthétiques traditionnelles de l'histoire de l'art ${ }^{20}$. Ils se perçoivent par effets de focale, par déclinaisons typologiques, comme un substrat dynamique, presque une matrice, où l'exceptionnel monumental n'apparait plus finalement qu'en tant que cas particulier. On pourrait parler d'architecture du banal, mais le terme a des connotations morales qui parasitent son sens. On pourrait utiliser le terme d'architecture régulière, dans le sens étymologique qu'utilise Michel Foucault, mais, en histoire de l'architecture, il tend à renvoyer au monumental. On pourrait parler d'architecture du quotidien, conforté en cela par les travaux de Michel De Certeau ${ }^{21}$, mais l'exceptionnel aussi a son quotidien. Mieux vaut alors parler d'architecture ordinaire, dans le double sens de l'épithète : à la fois banale et en ordre. L'ambivalence du terme doit cependant rester constamment à l'esprit de qui le manie.

L’ordinaire en architecture pose immédiatement le problème de son analyse, qui doit être à la fois qualitative et quantitative et doit par conséquent hiérarchiser ce qui relève de l'innovation et ce qui est de l'ordre de l'inertie. Ce faisant, l'analyse doit répondre, pour aboutir, à des hypothèses préalables, à des problématiques méthodiquement explicitées, qui obligent le chercheur à sortir des canevas préconçus et des catégories implicites, héritées de l'histoire de sa discipline. En d'autres termes, il y a une manière "exceptionnelle" et une manière "ordinaire" d'écrire l'histoire de l'art.

C'est à un renouvellement des rapports avec d'autres sciences de l'homme et de la société que nous invite l'analyse de l'ordinaire, en particulier avec l'histoire culturelle qui elle aussi traite autant des masses que des élites, et surtout avec l'archéologie qui analyse les multiples formes et temporalités de tout ce qui est resté là. C'est aussi à un réinvestissement de l'histoire quantitative qu'invite la construction de ce rapport à l'histoire culturelle, motivé en cela par la banalisation de nouveaux outils informatiques de spatialisation des données, et de spatialisation des

\footnotetext{
20. L'ouvrage de Bernard Rudofsky, L’architecture sans architecte. Brève introduction à l'architecture spontanée, 1964, Éditions du Chêne, Paris, 1977, est une figure imposée à tout propos consacré à l'historiographie de la banalité en architecture.

21. Michel de Certeau, Luce Giard, Pierre Mayol, L’invention du quotidien, 1980, nouvelle édition augmentée, Gallimard, Paris, 1990-1994, 2 volumes.
} 
questionnements, comme les systèmes d'information géographique (SIG) ${ }^{22}$. Les jeux d'échelles que mobilise pareil exercice nous invitent aussi à des incursions dans l'Alltagsgeschichte allemande, et surtout dans la microstoria italienne qui vise à écrire une histoire "vue d'en bas". Avec Edoardo Grendi, la micro-histoire a postulé l'intérêt de "l'exceptionnel normal", dans le sens où "le témoignage-document peut être exceptionnel parce qu'il évoque une normalité, une réalité si normale qu'elle demeure habituellement tue"23.

Comme le rappelle un récent numéro de la revue Perspectives ${ }^{24}$, les historiens d'art se sont parfois flagellés d'avoir trop longtemps cédé à la méthode monographique, et voient souvent leur salut dans la micro-histoire que nombre d'historiens pratiquent depuis trente ans. Bien que Carlo Ginzburg ait consacré à Piero della Francesca un ouvrage relevant de la micro-histoire25, la pratique de la monographie et la micro-histoire n'ont pas, a priori, de méthodologies ni d'objectifs communs. Importée en histoire de l'architecture contemporaine, la micro-histoire ne s'intéresserait pas seulement à tel ou tel architecte, mais, éventuellement, à tels ou tels artisans du bâtiment, réseaux de clientèle, sociétés professionnelles, modalités locales d'urbanisation, adaptations locales d'une technique constructive, réglementations de voirie, résistances à telle ou telle innovation, etc ${ }^{26}$. Idéalement, ces phénomènes sont ensuite à appréhender et à comparer à bien d'autres échelles. En tout état de cause, la micro-histoire qui séduit les historiens d'art, parce que son échelle est finalement celle à laquelle ils ont l'habitude de travailler, ne doit pas faire manquer l'approche et le traitement simultanés des grandes masses et des macro-phénomènes.

La micro-histoire aurait-elle refondé la monographie en histoire de l'art, ce qui reste sans doute à démontrer, elle ne nous porte certainement pas à espérer que soient revenus les temps où l’on ne s’intéressait qu'aux grands hommes ayant façonné leur époque ou œuvré à telle ou telle téléologie. Certes, il importe que l'histoire de l'art s'intéresse toujours à ces hommes-là, mais les chercheurs sont aussi attendus par tous les vaincus de l'histoire, la grande et les petites, par le “grouillement des disparus”, des “engloutis”, des “effacés”, à l’instar du Louis-François Pinagot

\footnotetext{
22. France Nerlich nous fait remarquer que les SIG pourraient bien être l'une des formes de cette écriture tridimensionnelle (voire quadridimensionelle) que nous appelons de nos voeux.

23. Edoardo Grendi, "Repenser la micro-histoire", in Jacques Revel (dir.), op. cit., p. 233-243. Article initialement publié dans Quaderni Storici, n 86, 1994, p. 539-549.

${ }^{24}$. Perspectives $\mathrm{n}^{\circ} 4,2006$, numéro thématique consacré à "La monographie d'artiste". Voir en particulier l'article de Pieter Uyttenhove, "Qu'importe qui conçoit ? Questionnement sur la monographie d'architecte”, p. 585-613.

25. Carlo Ginzburg, Enquête sur Piero della Francesca, Flammarion, Paris, 1983.

26. Sur ces questions, la bibliographie récente abonde, comme en témoignent les très nombreux titres inventoriés par Pieter Uyttenhove, art. cit.
} 
d'Alain Corbin ${ }^{27}$. Cette posture est particulièrement féconde pour l'histoire architecturale et urbaine des situations coloniales ${ }^{28}$.

S'il est sans doute intéressant de pratiquer une histoire de l'architecture écrite aussi bien "au ras du sol" que dans une ambition mesurée à la "macro-histoire", cette histoire doit être hiérarchisée et pondérée par un système d'échelles dont Jacques Revel rappelle que “c'est le principe de la variation qui compte, non le choix d'une échelle particulière" 29 . Un tel système d'échelle pourrait intégrer non pas seulement les faits "avérés", mais également les incertitudes, les malentendus (creative misunderstandings, au sens où l'entend Harold Bloom ${ }^{30}$ ), les résurgences de prime abord irrationnelles, ainsi que, dans la pratique, la partie documentable de l'arborescence des faits potentiels dont chaque situation du présent ne fit advenir qu'un seul. Un tel système d'échelle pourrait par exemple s'appliquer à l'histoire des normes du bâtiment, dans leurs emballements et leurs inerties, leurs rationalités et aberrations apparentes ou profondes, dans leur force incantatoire souvent inversement proportionnelle à leur efficacité ${ }^{31}$. Pour autant, cette histoire en échelles ne pourrait faire autrement qu'assumer et travailler son héritage historiographique majoritairement construit sur son rapport à l'histoire de l'élitaire et de la petite échelle, celle de l'œuvre d'art ou d'architecture. Quoi qu'il en soit, se pose à ce stade l'exposé le problème du statut du sujet et de l'acteur en histoire de l'art.

\section{Acteur et sujet}

"Le nouveau sujet possède à la fois surface et profondeur: l'individu tel qu'on le concevait autrefois, être raisonnable, rigoureux et réfléchi, était comme l'univers newtonien; le nouveau $[\ldots]$, sous certains rapports, se comporte comme un corpuscule en mouvement et, sous d'autres, comme une onde" ${ }^{32}$. En son temps, Lewis Mumford avait déjà relevé l'importance à traiter du banal comme du multiple, pour les œuvres comme pour les hommes. Plus près de nous, Pierre Bourdieu a démontré l'artificialité d'une historicisation du sujet fondée sur une rhétorique du moi, sur une sorte de philosophie de l'identité, qui deviennent le creuset des

\footnotetext{
27. Alain Corbin Le monde retrouvé de Louis-François Pinagot. Sur les traces d'un inconnu, 1798-1876, Flammarion, Paris, 1998.

${ }^{28}$. Il est utile en l'occurrence de poursuivre les rapprochements critiques entre l'histoire de l'architecture contemporaine et les études poscoloniales, notamment les subaltern studies, courant historique né en Inde qui développe une critique de l'historiographie nationaliste anticoloniale et qui vise à recouvrer les voix des vaincus de la décolonisation. Voir Achille Mbembe, "Qu'est-ce que la pensée postcoloniale?”, in Esprit, "Pour comprendre la pensée postcoloiale”, décembre 2006, p. 117-133 (propos recueillis par Olivier Mongin, Nathalie Empereur et JeanLouis Schlegel). Voir aussi Dipesh Chakrabarty, Provincializing Europe. Postcolonial thought and historical difference, Princeton University Press, Princeton, 2000 (cité par Achille Mbembe).

29. Jacques Revel, "Micro-analyse et construction du social", in Jacques Revel (dir.), op. cit., p. 19.

30. Harold Bloom, op. cit.

31. Voir les travaux de Jean-Pierre Épron, notamment Comprendre l'éclectisme, Norma, Paris, 1997, et ceux de Christian Moley, nortamment L'architecture du logement, culture et logique d'une norme héritée, Anthropos, Paris, 1998.

32. Lewis Mumford, "The Task of Modern Biography”, in English Journal, 23, 1934, p. 1-9, cité par Sabina Loriga, in Jacques Revel (dir.), op. cit., p. 227.
} 
téléologies. "Essayer de comprendre une vie, écrivait Bourdieu, comme une série unique et à soi suffisante d'événements successifs sans autre lien que l'association à un 'sujet' dont la constance n'est sans doute que celle d'un nom propre, est à peu près aussi absurde que d'essayer de rendre raison d'un trajet dans le métro sans prendre en compte la structure du réseau, c'est-à-dire la matrice des relations objectives entre les différentes stations." ${ }_{33}$

Michel Foucault affirmait déjà que "le discours [...] n'est pas la manifestation, majestueusement déroulée, d'un sujet qui pense, qui connaît, et qui le dit : c'est au contraire un ensemble où peuvent se déterminer la dispersion du sujet et sa discontinuité avec lui-même.”34 Parlant de "l'illusion ordinaire de l'unicité et de l'invariabilité", Bernard Lahire rappelle que l'unicité du soi n'est qu'un postulat de l'idéologie des sciences. Pour des raisons méthodologiques autant que morales, celle-ci se désintéresse des "lunatiques", des "girouettes", des “opportunistes" et des “caméléons"35, et traite prioritairement d'hommes supposés homogènes, fidèles à eux-mêmes et à l'intégrité de leur discours, qui entreverraient et donneraient à comprendre dans chacune de leurs paroles et de leurs actions les finalités de celles-ci. Ceux dont la cohérence n'atteindrait pas cette exemplarité, somme toute vasarienne ${ }^{36}$, seraient indignes d'histoire. Des micro-historiens comme Giovanni Levi manient de leur côté le concept de rationalité limitée ${ }^{37}$.

On pourrait rétorquer que ce qui intéresse avant tout les historiens d'architecture, ce sont moins les sujets que les œuvres où ils cherchent à repérer et construire des cohérences, qui sont ensuite à croiser avec celles que les contemporains ont reçues et gravées dans l'historiographie. Cependant, dans le domaine de l’œuvre (dont la notion pose par ailleurs problème), l’idéologie de l'unicité, et celle de l'unité, sont également battues en brèche depuis plus de quarante ans, notamment par des ouvrages théoriques aussi célèbres que ceux de Robert Venturi, Complexity and Contradiction in Architecture (1966) et Learning from Las Vegas (1972).

L'historien d'art a une propension à ériger en prophète de la réalité, et dans un second temps en grand ancêtre, l'artiste-héros dont la cohérence postulée des capacités interprétatives et

\footnotetext{
33. Pierre Bourdieu, "L'illusion biographique”, Actes de la Recherche en Sciences sociales, nº 62-63, juin 1986, p. 69-72.

34. Michel Foucault, L'archéologie du savoir, op. cit., p. 78. Voir aussi Michel Foucault, "Qu'est-ce qu'un auteur", in Bulletin de la Société française de philosophie, $\mathrm{n}^{\circ}$ 63, 1969, p. 73-104 ; Roland Barthes, "La mort de l'auteur", in Manteia, $\mathrm{n}^{\circ}$ 5, 1968, p. 12-17.

35. Bernard Lahire, L’homme pluriel. Les ressorts de l'action, Armand Colin / Nathan, Paris, 2001 (édition consultée : Hachette Littérature), p. 31.

36. Claude Mignot, "La monographie d'architecte à l'époque moderne en France et en Italie : esquisse d'historiographie comparée", in Perspectives, n 4, 2006, numéro thématique "La monographie d'artiste", p. 629-636. 37. Giovanni Levi, Le pouvoir au village. Histoire d'un exorciste dans le Piémont du XVIIe siècle, Turin, 1985, Gallimard, Paris, 1989. Cité par Jacques Revel (dir.), op. cit., p. 25.
} 
créatrices servirait mécaniquement la domination d'une "histoire des vanités"38 et de l'innovation. Quand bien même l'historien d'art se détournerait-il de cette figure, l'inertie des historiographies tendrait à l'y ramener sournoisement. Il est par ailleurs difficile au chercheur de renoncer à cette posture dans le cadre des enseignements universitaires, dans la mesure où les "grands ancêtres" ne peuvent être totalement inconnus des étudiants.

Y aurait-il, malgré tout, des manières moins univoques de définir les objets et construire les faits en histoire de l'art? Reconnaissons tout d'abord que la figure du héros n'est pas toujours le pain quotidien des historiens de l'architecture antique et médiévale, voire moderne, pour qui l'anonymat de l'artiste, faute de sources, est une contrainte, voire une matrice méthodologique. Pour la période contemporaine, l'apparition des images mécaniques écorne aussi la figure du héros. Celle-ci trouve d'autres limites lorsque des artistes contestent eux-mêmes leur statut d'artiste, ou lorsque des architectes mettent clairement en avant leur pratique quantitative ${ }^{39}$. Remarquons en passant que le héros n'est pas seulement le sujet, mais peut être aussi l'historien qui lui consacre une monographie ${ }^{40}$. Enfin, s'il n'est ignoré de personne que l'architecture est une pratique artistique et professionnelle fondamentalement collective, les architectes par stratégie personnelle de leur vivant ${ }^{41}$, et à leur suite leurs historiens, n'en attribuent pas moins les œuvres le plus souvent à leur seul nom. C'est autant une affirmation doctrinale (une œuvre, un nom) qu'une simplification de langage, tant serait longue la liste des collaborateurs à mentionner. C'est aussi une simplification de méthode qui unifie à bon compte l'architecture aux arts visuels.

La question fait évidemment toucher aux limites de l'histoire de l'art. L'exceptionnel et l'individuel sont des catégories inhérentes à la construction des objets et à la structuration de l'historiographie en histoire de l'art. Postuler l'importance de l'ordinaire et du sujet pluriel entraînerait-il pour autant la dilution de l'histoire de l'art, dans l'histoire d'un côté, dans l'archéologie de l'autre? Pour ce qui concerne l'architecture qui n'est pas qu'un art, et où l'appréhension de la quantité et du collectif a une valeur opératoire au moins équivalente à celle

\footnotetext{
38. L'expression est de June Komisar, «Looking at Collaboration as a Key to Creative Expression : The Case of Colonial Architecture », in Repenser les limites : l'architecture à travers l'espace, le temps et les disciplines/Changing Boundaries : Architectural History in Transition, conférence internationale organisée par l'INHA et la Society of Architectural Historians, septembre 2005. Les actes seront prochainement mis en ligne sur le site www.inha.fr 39. Voir en particulier "La monographie d'artiste, une contrainte, un modèle, un schéma adaptable?", débat entre Sylvie Aubenas, Éric de Chassey, Philippe Plagnieux, Agnès Rouveret, modéré par Michel Laclotte et Nabila Oulebsir, in Perspectives, "La monographie d'artiste", n 4, 2006.

${ }^{40}$. L'ouvrage que Jacques Le Goff consacra à la figure de Saint-Louis n'est pas l'exemple le moins célèbre, ni, sans doute, le moins paradoxal. Jacques Le Goff, Saint-Louis, Gallimard, Paris, 1996.

41. André Lurçat tria consciencieusement ses archives avant de les céder au CNAM, et oeuvra à construire sa fortune critique. Jean-Louis Cohen, André Lurcat (1894-1970), autocritique d'un moderne, Mardaga, Bruxelles, 1995. Pour ce qui concerne Henri Sauvage, il ne subsiste aucune trace directe du passage dans son agence de Robert Mallet-Stevens ou de Gabriel Guévrékian, dans les archives et les rares textes qu'il a laissés. Jean-Baptiste Minnaert, collectif IFA, The architectural drawings of Henri Sawvage - The works of an architect-decorator in the collections of the Institut Français d'Architecture and the Archives de Paris, Garland Publishing, New York, Londres, 1994, 2 vol. ; Jean-Baptiste Minnaert, Henri Sauvage, ou l'exercice du renouvellement, Norma, Paris, 2002.
} 
de la qualité et de l'individuel, la question est délicate. Pour ce qui concerne l'histoire des arts visuels, elle l'est sans doute davantage. L'intérêt d'une telle réflexion est d'élaborer des approches que nourrirait l'ensemble de la discipline.

Il y a quelques années, Jacques Lévy analysait ce "tournant géographique" qui marqua le dépassement de débats longtemps cristallisés en cette discipline autour de cinq antagonismes: physique ou humain, représentativité ou réalité, qualitatif ou quantitatif, particulier ou général, singulier ou universel. Ces clivages peuvent être transposés sous certaines conditions à l'histoire de l'architecture contemporaine, ne serait-ce que pour l'informer d'axes par lesquels la géographie est pleinement advenue, semble-t-il, à son statut de science sociale ${ }^{42}$. Assistons-nous à un tournant en histoire de l'architecture ? Une conférence internationale organisée à l'INHA en septembre 2005 a permis d'en visualiser des lignes de forces possibles ${ }^{43}$. Quoi qu'il en soit, les incursions dans d'autres sciences de l'homme et de la société, auxquelles nous nous sommes risqué ici, permettent de dégager les linéaments d'un programme collectif de recherche dont nous pourrions en conclusion énoncer quatre axes d'application.

$1^{\circ}$ Les interpénétrations de l'expertise, de la critique et de la recherche fondamentale, sur les plans socioprofessionnel, historiographique et méthodologique. Il serait fécond d'étudier les interactions entre les politiques patrimoniales, les politiques d'urbanisme, le mouvement des idées qui traversent l'histoire de l'architecture, trois registres où le chercheur est actant fragmentaire et multi-rationnel, dans un système d'acteurs dont il a une conscience partielle et au sein duquel ses visées intellectuelles sont évidemment liées à ses objectifs socioprofessionnels.

$2^{\circ}$ Les rapports de l'ordinaire à l'exceptionnel. Ceux-ci s'expriment, par exemple, dans la manière dont l'architecture ordinaire, patrimonialisée en Europe depuis une cinquantaine d'années par le fait des citoyens et des décideurs, infléchit les politiques urbanistiques et patrimoniales dans leur rapport à la monumentalité architecturale et urbaine, et renouvelle en retour les méthodes et les corpus de l'histoire de l'architecture.

$3^{\circ}$ Les modalités d'invention des objets en histoire de l'art. Celles que peuvent pister, par exemple, une réflexion sur l'invention de l'archive et un questionnement sur l'horizon d'attente de l'élection d'un phénomène au statut d'objet scientifique. Ainsi l'invention de l'archive locale at-elle déterminé le renouvellement de l'historiographie de l'histoire de l'architecture, de l'urbanisme et du patrimoine en situation coloniale.

\footnotetext{
42. Jacques Lévy, "L'exception ordinaire”, dans Jacques Lévy, Michel Lussault (dir.), Logiques de l'espace, esprit des lieux. Géographies à Cerisy, Belin, Paris, 2000, p. 333-343. Voir aussi Jacques Lévy, Le tournant géographique. Penser l'espace pour lire le monde, Belin, Paris, 1999.

43. Repenser les limites..., colloque cité.
} 
$4^{\circ}$ Les interactions, transferts et ruptures dans les pratiques et les représentations des acteurs de la production du bâti. En particulier les circulations de modèles et les malentendus, sur lesquels ces représentations et ces pratiques se sont effectuées dans la production architecturale et dans l'écriture de son histoire au XXe siècle.

Ce programme est modestement ambitieux. Mais il semble que l'histoire de l'art en général, l'histoire de l'architecture contemporaine en particulier, soient engagées dans un processus de réinvention dont il serait intéressant de rendre compte.

\section{Jean-Baptiste Minnaert}

Né en 1964, Jean-Baptiste Minnaert est professeur d'histoire de l'art contemporain à l'université François Rabelais, Tours, et membre de l'équipe de recherche InTRu (Interactions, transferts, ruptures artistiques et culturels). Ses travaux portent sur des questions d'histoire de l'architecture et de l'urbanisme au XXe siècle. Il a publié une monographie d'architecte, Henri Sauvage, ou l'exercice du renouvellement (Norma, 2002) et a dirigé un ouvrage collectif, Histoires d'architectures en Méditerranée XIXe-XXe siècles. Écrire l'bistoire d'un héritage bâti (Éditions de la Villette, 2005). 